\title{
LETTERS
}

\section{Mediation of the solar wind termination shock by non-thermal ions}

\author{
R. B. Decker ${ }^{1}$, S. M. Krimigis ${ }^{1,2}$, E. C. Roelof ${ }^{1}$, M. E. Hill ${ }^{1}$, T. P. Armstrong ${ }^{3}$, G. Gloeckler ${ }^{4}$, D. C. Hamilton ${ }^{5}$ \\ \& L. J. Lanzerotti ${ }^{6,7}$
}

\begin{abstract}
Broad regions on both sides of the solar wind termination shock are populated by high intensities of non-thermal ions and electrons. The pre-shock particles in the solar wind have been measured by the spacecraft Voyager 1 (refs 1-5) and Voyager 2 (refs 3 , 6). The post-shock particles in the heliosheath have also been measured by Voyager 1 (refs 3-5). It was not clear, however, what effect these particles might have on the physics of the shock transition until Voyager 2 crossed the shock on 31 August-1 September 2007 (refs 7-9). Unlike Voyager 1, Voyager 2 is making plasma measurements ${ }^{7}$. Data from the plasma ${ }^{7}$ and magnetic field ${ }^{8}$ instruments on Voyager 2 indicate that non-thermal ion distributions probably have key roles in mediating dynamical processes at the termination shock and in the heliosheath. Here we report that intensities of low-energy ions measured by Voyager 2 produce non-thermal partial ion pressures in the heliosheath that are comparable to (or exceed) both the thermal plasma pressures and the scalar magnetic field pressures. We conclude that these ions are the $>0.028 \mathrm{MeV}$ portion of the non-thermal ion distribution that determines the termination shock structure ${ }^{8}$ and the acceleration of which extracts a large fraction of bulk-flow kinetic energy from the incident solar wind ${ }^{7}$.
\end{abstract}

We report $>0.028 \mathrm{MeV}$ ion and $>0.022 \mathrm{MeV}$ electron energy measurements made by the Voyager 2 Low Energy Charged Particle $^{10}$ (LECP) instrument in the solar wind, at the termination shock, and in the heliosheath during 2005-2008. We refer to the first crossing of the termination shock by Voyager 2, on day 242 of 2007, as TS-1 (refs 7,8$)$.

Figure $1 \mathrm{~b}$ shows that the large energy-dependent variations of lowenergy ion intensities measured in the solar wind diminish within 7-8 days of TS-1 occurring. Smaller variations continue in the heliosheath until day 342 of 2007 (2007.94), when a possible transient disturbance reduced intensities of higher energy ions, causing large variations in the spectral index $\gamma(E)$ (Fig. 1c). The low-energy ion energy spectrum measured so far by Voyager 2 in the heliosheath is even flatter than that measured by Voyager 1. For the 123-day period following TS-1 (days 242 to 365 of 2007), Voyager 2 data are well fit by a single power law with index $\gamma=-1.24 \pm 0.05$. By contrast, for the 160-day period following the crossing of the termination shock by Voyager 1 (day 352 of 2004 to day 144 of 2005), that spacecraft's data are well fit by a single power law with index ${ }^{4} \gamma=-1.67 \pm 0.03$, which later increased slightly ${ }^{6}$ to $\gamma \approx-1.4$ to -1.6 .

The salient feature in the LECP measurements of TS- 1 is that the non-thermal partial pressure produced by $0.028-3.5 \mathrm{MeV}$ energetic ions in the heliosheath is comparable to (or exceeds) both the thermal plasma pressure ${ }^{7}(n k T)$ and the scalar pressure $\left(B^{2} / 8 \pi\right)$ of the magnetic field ${ }^{8}$. The very flat ion spectrum recorded by Voyager 2 enhances the suprathermal partial ion pressure, which is a lower limit on the total particle pressure. The partial pressure $>0.028 \mathrm{MeV}$ is compared in Fig. 1a with that measured by Voyager 1 . The horizontal dashed line shows the magnetic field pressure calculated using the Voyager 1 mean heliosheath field intensity (at the time of writing, Voyager 2 magnetic field data were unavailable beyond day 245 of 2007). The Voyager 1 partial ion pressure is generally comparable to or exceeds the mean field pressure in the heliosheath. The Voyager 2 partial ion pressure is almost always greater than that measured by Voyager 1 (especially in the pre-shock region). The termination shock and heliosheath regions show high intensities of energetic electrons (Fig. 1d), which were also observed by Voyager 1. The high electron intensities imply that electron impact ionization can have a role in the generation of suprathermal pickup hydrogen that is comparable to (or greater than) that of charge-exchange ionization by the solar wind.

Except for increases due to passage of a merged interaction region and the associated interplanetary shock in March 2006 (ref. 11), intensities of ions $<0.2-0.3 \mathrm{MeV}$ in energy remained near background (Fig. 2a, b) until about day 210 of 2007, 32 days before TS1. This was not the case for Voyager 1, where intensities of 0.0417.6 MeV ions, averaged over tens of days, increased steadily from mid-2002 to the end of 2004 (refs 4-6). Relatively large intensity increases of $0.14-0.22 \mathrm{MeV}$ ions were recorded by Voyager 1 during the second half of 2002 and during 2004. For ion energies $>0.2-$ $0.3 \mathrm{MeV}$ (Fig. 2c-e), upstream intensities are comparable for Voyagers 1 and 2, as Fig. 2e shows; however, over comparable time durations following their respective termination shock crossings, downstream heliosheath intensities observed so far by Voyager 2 are higher than those observed by Voyager 1. Increases in relativistic electrons (Fig. 2f) upstream are less frequent and less intense for Voyager 2 than for Voyager 1, as is true for $<0.2-0.3 \mathrm{MeV}$ ions; but downstream they are more intense for Voyager 2 than for Voyager 1 , as is true for $>0.2-0.3 \mathrm{MeV}$ ions. The variation of the $>70 \mathrm{MeV}$ proton count rate (Fig. $2 \mathrm{~h}$ ) indicates modulation of highenergy ion intensities, probably due to solar cycle variations and to passage of transient disturbances, such as the Forbush decrease in March 2006 that was associated with a merged interaction region.

Termination shock ions measured by Voyager 1 in the upstream solar wind between mid-2002 and the end of 2004 were highly anisotropic; they were beamed mainly anti-sunwards along the nearly azimuthal spiral interplanetary magnetic field, that is, in the $-\mathrm{T}$ direction ${ }^{1,3-5}$. Those measured by Voyager 2 in the upstream solar wind between the beginning of 2005 and day 242 of 2007 were also anisotropic, but beamed mainly sunwards along the spiral interplanetary magnetic field, that is, in the $+\mathrm{T}$ direction, as Fig. $2 \mathrm{~g}$ shows $^{3,6}$. However, in both the Voyager 2 and Voyager 1 data, anisotropies in 
the heliosheath are reduced relative to those in the solar wind, except for the two oppositely directed bursts recorded by Voyager 2 on 2007.9 and 2008.0 in Fig. 2 g.

The measurements of high partial pressures of low-energy ions and of high intensities of high-energy electrons made using the Voyager 2 LECP instrument have immediate implications for the nature and processes of the termination shock, the foreshock and the heliosheath. These in turn have ramifications for the global structure of the heliosphere, particle acceleration and propagation processes, as well as for the collisionless shock structure. Voyager 1 entered the termination foreshock proper at a helioradius of $85.2 \mathrm{AU}$ ( $1 \mathrm{AU}$ is the Sun-Earth distance), and Voyager 2 did so at a helioradius of $75.3 \mathrm{AU}$, roughly $10 \mathrm{AU}$ nearer the Sun than Voyager 1. First detection by Voyager 2 of termination shock particles around the time that the shock swept over Voyager 1 during its inward movement is qualitatively consistent with three-dimensional heliosphere models which predict an asymmetric termination shock that is nearer the Sun at the position of Voyager 2 because of symmetry-breaking effects of the interstellar magnetic field ${ }^{12,13}$. However, quantitative issues are still under examination ${ }^{14}$.

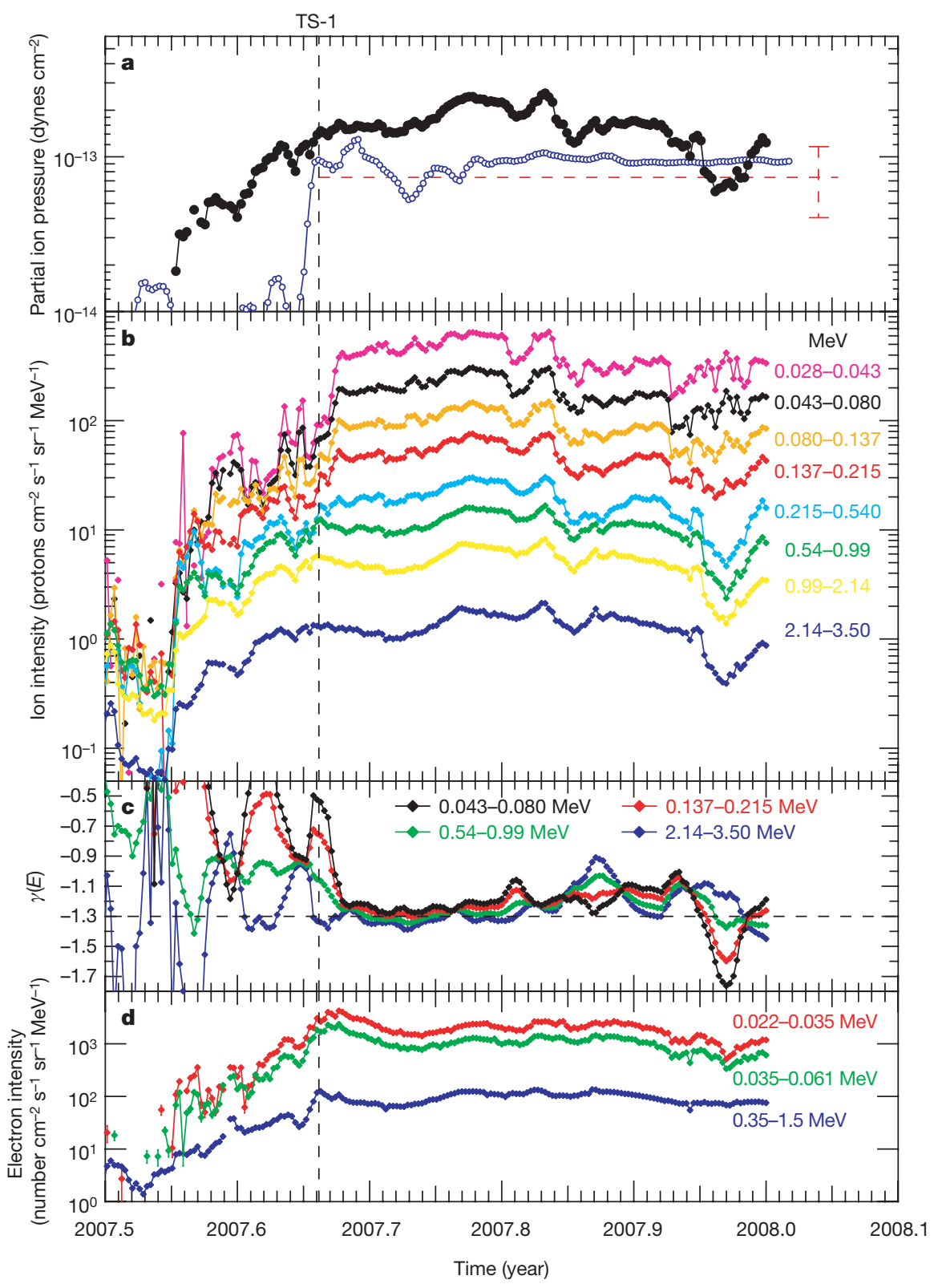

Figure 1 | Low-energy ions and electrons measured by Voyager 2 near the termination shock during 2007. Voyager 2 crossed the termination shock at least five times during days 242 to 244 of 2007 (refs 7-9) at helioradius $R=83.65 \mathrm{AU}$ and heliographic latitude $L=-27.5^{\circ}$. a, The black trace shows Voyager $20.028-3.5 \mathrm{MeV}$ partial ion (proton) pressures. The blue trace shows Voyager $10.040-4.0 \mathrm{MeV}$ partial proton pressures, time-shifted so that the termination shock crossing of Voyager 1 on day 351 of 2004 coincides with TS-1. Both traces exceed the magnetic field pressure $P_{B_{1}}=B_{1}{ }^{2} / 8 \pi$ (dashed red line), calculated using the mean heliosheath field intensity $B_{1}=0.123 \pm 0.035 \mathrm{nT}$ ( \pm 1 s.d.) measured by Voyager 1 between days 1 and 110 of 2005 (ref. 20). The upper and lower bounds of the vertical error bar are $P_{B_{1}}$ evaluated respectively at $B_{1}=0.158$ and $0.088 \mathrm{nT}$. The
Voyager 1 and 2 ion pressures were calculated using intensities of ions arriving from the sunwards and anti-sunwards directions, to reduce contributions from field-aligned beams that arrive mainly from the azimuthal direction. $\mathbf{b}$, Intensities in the eight Voyager 2 ion channels calculated using proton energy passbands and efficiencies. c, Differential spectral index $\gamma(E)$ evaluated at the logarithmic means of the energy passband for the channels indicated ${ }^{6} . \mathbf{d}, 0.022-1.5 \mathrm{MeV}$ electron intensities; ordinate of blue trace is multiplied by 30 . Electron intensities peak at or near the termination shock, are nearly isotropic in the solar wind and heliosheath, and at $\sim 0.03 \mathrm{MeV}$ and $\sim 0.7 \mathrm{MeV}$ are respectively higher by a factor of $\sim 4$ or lower by a factor of $\sim 3$ than intensities of ions at the same energy. All data shown are one-day averaged; data in $\mathbf{c}$ are also five-point smoothed. 


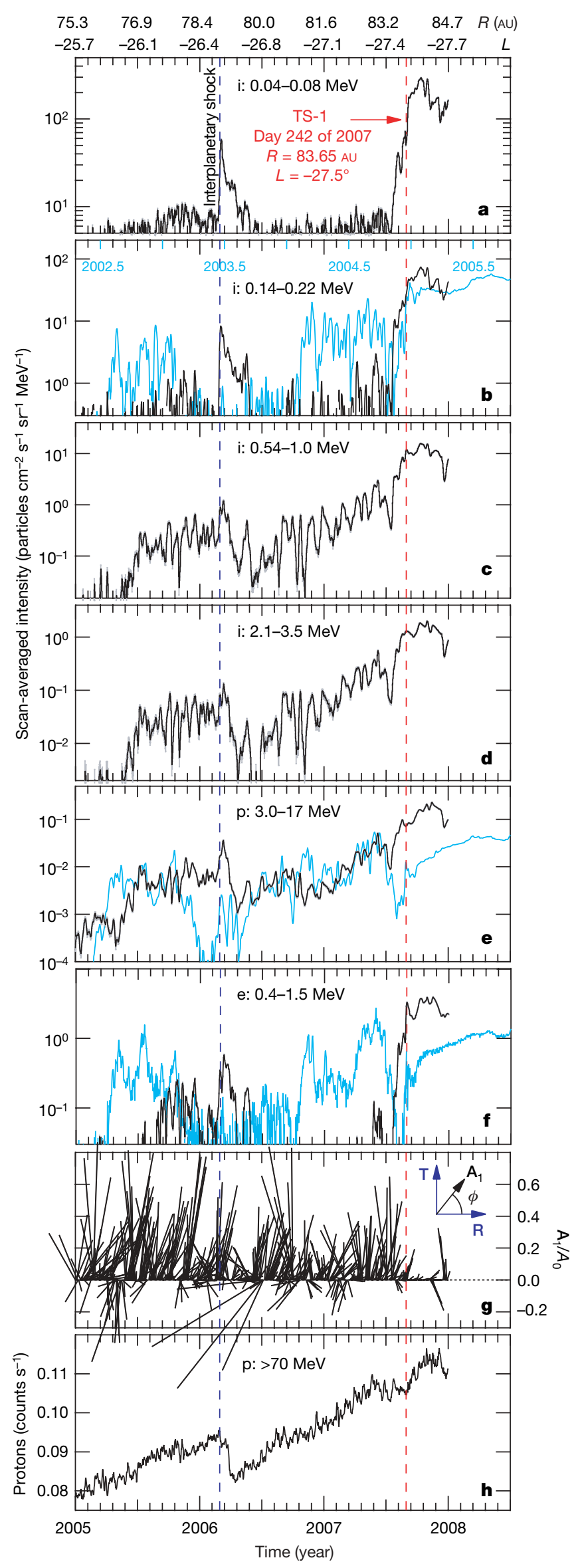

Numerical models had predicted that Voyager 2 should observe termination shock ion anisotropies directed towards the Sun (the $+\mathrm{T}$ direction), in the opposite direction to those observed by Voyager 1 (ref. 12), which is consistent with the data. Currently, the models do not explain why the intensities of termination shock ions observed by Voyager 2 in the solar wind are deficient at energies $<0.2-0.3 \mathrm{MeV}$ until only $\sim 30$ days before TS- 1 . One possibility is that shock normal vectors in regions of the termination shock to which Voyager 2 was magnetically connected were, on average, more nearly perpendicular to the field than were those to which Voyager 1 was connected, thus hindering the upstream escape of slower, lower energy ions from the termination shock, and reducing their intensities at Voyager 2 (ref. 15). Another possibility, suggested by recent modelling of termination shock motion ${ }^{16}$, is that during the $\sim 2.5 \mathrm{yr}$ periods when upstream termination shock particles were observed at the spacecraft, Voyager 1 remained relatively close to the termination shock in comparison with Voyager 2. However, the intensities of 0.35$1.5 \mathrm{MeV}$ electrons ( speeds $\sim 160 \mathrm{AU} \mathrm{d}^{-1}$ ) in the solar wind measured by Voyager 2 also are lower than those measured by Voyager 1 (Fig. 2f). This might suggest that the electron source region is more remote from the spacecraft than is that of the termination shock ions.

Suprathermal ions in the heliosheath probably originate as solar wind pickup ions that undergo acceleration or heating, or both, at the termination shock and in the heliosheath ${ }^{17-19}$. In the simplest application of diffusive shock acceleration theory, a spectral index of $\gamma \approx-1.25$ for $0.028-3.5 \mathrm{MeV}$ heliosheath ions implies a density compression ratio of $\sim 3$ across the termination shock, which is inconsistent with the value of $\sim 2$ deduced from Voyager 2 plasma and field data $^{7,8}$. This spectral index is also inconsistent with the value of $\gamma=-1.5$ predicted when the suprathermal tail of the solar wind pickup protons is heated across the termination shock ${ }^{19}$. We note that these current theories and models are applicable under conditions that are steady in time and uniform in space. Voyager 2 is still evidently in the region of the heliosheath near the termination shock, and it is not clear from the Voyager 2 data that either of the conditions is met. More importantly, we stress that the estimated partial ion pressure derived from the LECP data in Fig. 1a considerably exceeds the magnetic pressure and is consistent with other Voyager 2 plasma $^{7}$ and magnetic field ${ }^{8}$ data which indicate that energized

Figure 2 | Energetic ions and electrons measured by Voyager 2 during 2005-2007. Expanded view showing Voyager 2 LECP data during 2005-2007, when Voyager 2 moved from $R=75.3 \mathrm{AU}, L=-25.7^{\circ}$ to $R=84.7 \mathrm{AU}, L=-27.7^{\circ}$. Instruments on Voyager 1 began measuring high intensities of termination shock particles upstream of the shock in mid 2002 at $R=85.2 \mathrm{AU}, L=33.8^{\circ}$ (refs 1,2$)$. Voyager 1 crossed the termination shock at $R=94.0 \mathrm{AU}$ on day 351 of 2004 (refs $3-5,20,21$ ) as the shock moved radially inwards as a result of decreasing solar wind ram pressure ${ }^{22}$. The asymmetry of the termination shock allowed Voyager 2, then in the solar wind at only $R=75.3 \mathrm{AU}, L=-25.6^{\circ}$, to enter the termination foreshock, where the Voyager 2 instruments began measuring termination shock particles $^{3,6}$. a-f, Black traces are Voyager 2 LECP scan-averaged intensities at indicated particle energies ( $i$, ion; $p$, proton; e, electron); grey error bars show 2 s.d. Blue traces $(\mathbf{b}, \mathbf{e}, \mathbf{f})$ are Voyager 1 LECP intensities shifted in time by $+2.705 \mathrm{yr}$ to align the termination shock crossings of Voyager 1 (day 351 of 2004) and Voyager 2 (day 242 of 2007); Voyager 1 data shown cover the period between day 109 of 2002 and day 291 of 2005 . The time (in years) for the Voyager 1 data are indicated along the top axis of $\mathbf{b}$. $\mathbf{g}$, First-order anisotropy vector $\mathbf{A}_{1} / A_{0}$ derived from a least-squares Fourier fit in the $\mathrm{R}-\mathrm{T}$ plane (unit vector $\mathbf{R}$ points radially outward from Sun, unit vector $\mathbf{T}$ points in the direction of normal planetary motion) of measured intensities in seven of eight $45^{\circ}$ sectors for proton intensity in $\mathbf{e}^{4,5}$. The $\mathbf{A}_{1} / A_{0}$ 'whiskers' point in the direction in which the particles are moving, and $\phi$ is the azimuthal angle in the $\mathrm{R}-\mathrm{T}$ plane $\mathrm{e}^{4} . \mathrm{h}$, Count rate of $>70 \mathrm{MeV}$ protons. The rapid decrease followed by partial recovery at the end of 2007 suggests that similar intensity decreases seen in all LECP channels are caused by passage of a transient disturbance through the heliosheath. Voyager 2 helioradius $R$ and heliographic latitude $L$ are indicated at half-year intervals along the top of a. All data shown are one-day averaged and five-point smoothed. 
pickup ions (as measured by the LECP instrument) have a major dynamical role in mediating the termination shock structure and its variability, thereby significantly affecting dynamical processes in the heliosheath plasma ${ }^{7,8}$.

\section{Received 21 February; accepted 15 April 2008.}

1. Krimigis, S. M. et al. Voyager 1 exited the solar wind at a distance of $\sim 85$ AU from the Sun. Nature 426, 45-48 (2003).

2. McDonald, F. B. et al. Enhancements of energetic particles near the heliospheric termination shock. Nature 426, 48-51 (2003).

3. Stone, E. C. et al. Voyager 1 explores the termination shock region and the heliosheath beyond. Science 309, 2012-2020 (2005).

4. Decker, R. B. et al. Voyager 1 in the foreshock, termination shock, and heliosheath. Science 309, 2020-2024 (2005).

5. Krimigis, S. M. et al. in Connecting Sun and Heliosphere (Proc. Solar Wind 11/SOHO 16 Conf.) (eds Fleck, B., Zurbuchen, T. H. \& Lacoste, H.) 21-28 (ESA, Noordwijk, 2005).

6. Decker, R. B. et al. in Physics of the Inner Heliosheath: Voyager Observations, Theory, and Future Prospects (Proc. 5th IGPP Internat. Astrophys. Conf.) (eds Heerikhuisen J., Florinski, V., Zank, G. P. \& Pogorelov, N. V.) 73-78 (AIP, Melville, New York, 2006).

7. Richardson, J. D. et al. Cool heliosheath plasma and deceleration of the upstream solar wind at the termination shock. Nature doi:10.1038/nature07024 (this issue).

8. Burlaga, L. F. et al. Magnetic fields at the solar wind termination shock. Nature doi:10.1038/nature07029 (this issue).

9. Gurnett, D. A. \& Kurth, W. S. Intense plasma waves at and near the solar wind termination shock. Nature doi:10.1038/nature07023 (this issue).

10. Krimigis, S. M. et al. The Low Energy Charged Particle (LECP) experiment on the Voyager spacecraft. Space Sci. Rev. 21, 329-354 (1977)

11. Richardson, J. D. et al. Source and consequences of a large shock near $79 \mathrm{AU}$. Geophys. Res. Lett. 33, L23107 (2006).

12. Opher, M., Stone, E. C. \& Liewer, P. C. The effects of a local interstellar magnetic field on Voyager 1 and 2 observations. Astrophys. J. 640, L71-L74 (2006).
13. Pogorelov, N. V. in Physics of the Inner Heliosheath: Voyager Observations, Theory, and Future Prospects (Proc. 5th IGPP Internat. Astrophys. Conf.) (eds Heerikhuisen J., Florinski, V., Zank, G. P. \& Pogorelov, N. V.) 3-13 (AIP, Melville, New York, 2006).

14. Pogorelov, N. V., Stone, E. C., Florinski, V. \& Zank, G. P. Termination shock asymmetries as seen by the Voyager spacecraft: The role of the interstellar magnetic field and neutral hydrogen. Astrophys. J. 668, 611-624 (2007).

15. Jokipii, J. R., Giacalone, J. \& Decker, R. B. Energy spectra of energetic particles upstream of the termination shock. Eos 88 (Fall meeting), abstr. SH11A-07 (2007).

16. Washimi, H. et al. A forecast of the heliospheric termination-shock position by three-dimensional MHD simulations. Astrophys. J. 670, L139-L142 (2007).

17. le Roux, J. A., Webb, G. M., Florinski, V. \& Zank, G. P. A focused transport approach to pickup ion shock acceleration: Implications for the termination shock. Astrophys. J. 662, 350-371 (2007).

18. Giacalone, J. \& Jokipii, J. R. Energetic particles around the termination shock: Numerical simulations for a blunt shock with cross-field diffusion. Astrophys. J. 649, L137-L140 (2006).

19. Fisk, L. A., Gloeckler, G. \& Zurbuchen, T. H. Acceleration of low-energy ions at the termination shock of the solar wind. Astrophys. J. 644, 631-637 (2006).

20. Burlaga, L. F. et al. Crossing the termination shock into the heliosheath: magnetic fields. Science 309, 2027-2029 (2005).

21. Gurnett, D. A. \& Kurth, W. S. Electron plasma oscillations upstream of the solar wind termination shock. Science 309, 2025-2027 (2005).

22. Richardson, J. D. et al. Relation between the solar wind dynamic pressure at Voyager 2 and the energetic particle events at Voyager 1. J. Geophys. Res. 110, A09106 (2005).

Acknowledgements Work at the Johns Hopkins University Applied Physics Laboratory was supported by the Voyager Interstellar Mission under NASA grant NNX07AB02G

Author Information Reprints and permissions information is available at www.nature.com/reprints. Correspondence and requests for materials should be addressed to R.B.D. (robert.decker@jhuapl.edu). 